\title{
Genesis
}

Manuscrits - Recherche - Invention

45 | 2017

Hugo

Catherine Viollet et Danielle Constantin (dir.), Genre, sexes, sexualités. Que disent les manuscrits autobiographiques?, Presses universitaires de Rouen et du Havre, coll. " Genres à lire... et à penser ", 2016, 159 p.

\section{Véronique Montémont}

\section{OpenEdition}

Journals

Édition électronique

URL : http://journals.openedition.org/genesis/3069

DOI : 10.4000/genesis.3069

ISSN : 2268-1590

Éditeur :

Presses universitaires de Paris Sorbonne (PUPS), Société internationale de génétique artistique littéraire et scientifique (SIGALES)

\section{Édition imprimée}

Date de publication : 5 décembre 2017

Pagination : 201-202

ISBN : 979-10-231-0580-3

ISSN : 1167-5101

Référence électronique

Véronique Montémont, «Catherine Viollet et Danielle Constantin (dir.), Genre, sexes, sexualités. Que disent les manuscrits autobiographiques? Presses universitaires de Rouen et du Havre, coll. "Genres à lire... et à penser », 2016, 159 p. », Genesis [En ligne], 45 | 2017, mis en ligne le 26 novembre 2018, consulté le 12 janvier 2021. URL : http://journals.openedition.org/genesis/3069; DOI : https://doi.org/ 10.4000/genesis.3069 
Catherine Viollet et Danielle Constantin (dir.), Genre, sexes, sexualités. Que disent les manuscrits autobiographiques?, Presses universitaires de Rouen et du Havre, coll. «Genres à lire... et à penser», 2016, 159 p.

Compte rendu par Véronique Montémont*

Genre, sexes, sexualités, sous-titré Que disent les manuscrits autobiographiques? est une passionnante exploration dans les écritures de soi, du XIXe au XX ${ }^{\mathrm{e}}$ siècle, qui combine études de genre, problématiques historico-sociales et approche génétique des textes. À travers l'examen des manuscrits, des versions successives, ou encore par la lecture croisée d'œuvres nées du même substrat autobiographique, Catherine Viollet, qui avait initié ce volume, et Danielle Constantin, qui en a poursuivi et mené à terme la publication, démontrent de manière pertinente que la triple question du genre, du sexe et de la sexualité, fondatrice de l'identité d'un individu, est aussi l'un des lieux de conflit les plus vifs entre la personne et la société - et de ces heurts, les écrits personnels et leurs manuscrits plus que les autres portent la trace. En effet, souvent soustraits au regard public, par choix ou par nécessité, les journaux, les premières versions des œuvres, les textes posthumes témoignent de «conflits, d'enjeux, d'ambiguïtés et de contradictions, d'autocensure et de censure, d'ajustement entre reproduction et subversion des normes » (D. Constantin), tous phénomènes que le volume s'assigne la tâche d'éclairer.

Cet ambitieux programme est servi par un corpus d'études aussi varié que pertinent, qui met en lumière la violence répressive, qu'elle soit morale, légale ou psychologique, qu'exercent les assignations en matière de genre social ou de sexualité. Il éclaire ainsi la question du poids de ces dernières sur les identités, qui forme le thème de la première partie de l'ouvrage. Le texte liminaire de Marion Krauthaker, consacré à Mes souvenirs de l'hermaphrodite Herculine Barbin, retrace ainsi le parcours douloureux de celui/celle qui est né.e dans un corps aux attributs biologiques à la fois féminins et masculins. Eduqué.e comme une fille, Herculine, après avoir subi la violence sociale et médicale, se voit réassigner une identité masculine : ses Souvenirs, entre mémoires et lettre de suicide, sont le seul espace où sortir d'un «corps et d'une individualité aliénés face à l'impossibilité de l'existence sociale». Autre destinée tragique, retracée par Mateusz Chmurski, celle de József Brenner, dit Géza Csáth (1887-1919), médecin, journaliste, musicien et diariste hongrois. Cet homme surdoué et tourmenté, qui projette d'écrire un grand roman, sombre rapidement dans la dépendance à la morphine; après avoir assassiné sa femme, il se suicide. Son journal personnel - plus de vingt cahiers - permet de comprendre comment Csáth a utilisé l'écriture pour se réinscrire dans un corps dispersé, traversé par la pulsion toxicomaniaque et le désir sexuel exacerbé; le journal, inventaire obsessionnel du fonctionnement corporel, devient un «cadre identitaire, un espace capable d'agréger quasiment tout ce qui le concernait» pour former un «textecorps ». Chez Yves Navarre, dont Sylvie Lannegrand étudie «L'écriture comme "acte sensuel" », la contradiction réside entre une homosexualité assumée et vécue,

\section{Comptes rendus d'ouvrages}

et la réduction de la figure publique de l'écrivain à cette composante identitaire. Cette tenaille est analysée à la faveur d'une lecture contrastive du journal inédit et de certaines œuvres publiées : on y voit comment Navarre, tenant d'une écriture de la «sensualité», bien plus que de celle d'une sexualité gaie, se sent marginalisé au sein de sa propre communauté. Au bout du compte, seule l'écriture, dispositif de survie, «est capable de conférer le sentiment d'identité dont l'auteur a besoin» : c'est aux lecteurs, aux critiques, que Navarre s'en remet pour accepter toutes les facettes de sa personnalité - et il sera souvent déçu.

Si Annie Ernaux ne vit pas de manière aussi conflictuelle l'expression des affects et du désir, il n'en demeure pas moins que la mise en forme de leur expression littéraire demeure pour elle un point problématique. Julie LeBlanc révèle, à partir d'une analyse des brouillons de Passion simple, comment la narratrice a expérimenté plusieurs ordonnances, à la recherche d'un «début qui crève la page, et place [s]a voix, sexe ou mort». La comparaison génétique dévoile le travail opéré pour dépassionner, du moins en apparence, les dimensions érotiques, pornographiques, désirantes, de sorte à trouver une forme littéraire apte à rendre dicible l'expérience extrême de la passion.

La deuxième partie du volume porte sur une autre ligne de conflit entre identité genrée, orientation sexuelle, érotisme et société : celle de la censure. Selon que des pages sont ou non destinées à la publication, selon aussi le degré jusqu'auquel on a intériorisé certains interdits, on peut lire dans les écritures personnelles l'expression

* Université de Lorraine. 
d'une contradiction récurrente entre l'envie de dire et la peur du dévoilement, jusqu'à l'autocensure. C'est ainsi que Kimberley Page-Jones relit les Carnets de Coleridge, analysant comment celui-ci déplace l'expression du désir, dans des pages où s'encrypte la lutte entre affect et refoulement, parfois à l'aide du dessin, «à la fois esquisse et biffure», qui «matérialise l'ambiguïté du désir». Nicole Cadène, elle, revient sur le journal de Marie-Edmée Pau, artiste lorraine (1845-1871) et diariste. Sans souffrir, comme Herculine Barbin, d'un décalage entre son corps et son genre social, cette artiste ne se reconnaît pas pour autant dans le modèle féminin de l'époque, sur un double plan sexuel et social : il lui faudrait pour cela renoncer à ses ambitions artistiques. Pour autant, elle reste violemment imprégnée de morale catholique, ce qui transforme son amitié amoureuse avec Marie-Paule Courbe, dite «Mary», en violente tension intérieure. Selon la belle formule de Nicole Cadène, ce conflit qu'exprime le journal entre une morale rigide, dont est imprégnée l'éducation des femmes à la fin du XIXe siècle, et la violence des passions «éclair[e] de [son] faisceau le sombre océan des rapports de genre». Pour Anaïs Nin, qui tint son journal durant toute sa vie, la censure s'opère entre les versions publiées (même si l'une d'elles est prétendument non expurgée) et le manuscrit. Dans celui-ci, qu'étudie avec précision Simon Dubois Boucheraud, Nin analyse sans fard la complexité de ses désirs, qu'il s'agisse d'érotisme, de maternité, de rôles affectifs ambivalents (l'amant dont on se sent mère tout en souhaitant, aussi, être la fille) jusqu'à l'exposé d'un inceste paternel consenti. «L'amour entre un homme et une femme n'est que résistance et conflit», écrit Nin; mais elle choisit d'aller résolument vers l'exploration de sa sensualité, aussi transgressive soit-elle, dont elle réinvestira certains motifs, de manière expurgée, dans une nouvelle. C'est également à une lecture comparative que nous invite l'étude d'Émeline André, consacrée au parcours génétique du Journal de guerre, de Simone de Beauvoir, dont des éléments ont été réinvestis dans Deuxième Sexe et La Force des Choses. Des mécanismes d'autocensure sont à l'œuvre, visant notamment à gommer l'aveu de certaines relations, homosexuelles ou hétérosexuelles au sein d'un triangle amoureux. L'article met en évidence le caractère stratégique, et même politique, de l'opération : si Beauvoir ne redoute aucunement le jugement moral, elle cherche à préserver sa légitimité d'essayiste, en refusant de fournir des armes à ses contempteurs. «L'autocensure participe ainsi à la mise en œuvre d'une représentation contrôlée, [...] largement conditionnée par la réception de son essai.»C'est enfin à Jack Kerouac que Danielle Constantin consacre le dernier article de cette deuxième partie, dépliant avec minutie les récritures et suppressions exigées par les éditeurs préalablement à la publication de Sur la route, dans le contexte d'une Amérique puritaine, sexiste et homophobe : on voit à quel point le texte a perdu de son audace, de sa force et de sa qualité, par un gommage et un affadissement systématiques de sa composante sexuelle et homosexuelle.

La troisième partie de l'ouvrage, enfin, rassemble quatre études de la genèse de l'œuvre de Violette Leduc, dont Catherine Viollet présente d'abord la relation d'autorité ambiguë qui la liait à Simone de Beauvoir, laquelle publia de manière posthume La Chasse à l'Amour, en l'amputant d'un tiers. Des choix éditoriaux étudiés de plus près par Mireille Brioude qui montre comment, dans le cahier 6, le travail conjoint de Leduc et Beauvoir a modifié profondément la scène du premier rapport sexuel avec l'amant, lui ôtant son caractère poétique et «contemplatif» au profit d'une relation plus événementielle, et au prix d'une a(du)ltération de la qualité littéraire. Anaiis Frantz, elle, fait émerger une véritable «poétique de la bâtardise», dont l'une des matérialisations est un lapsus qui mêle figure de la mère et masculinité. Alison Peron, qui revient sur la censure de La Chasse à l'amour, montre enfin comment Leduc, dans ses manuscrits, travaille l'ambiguïté et la porosité du masculin / féminin, un «trouble dans les genres grammaticaux en relation avec le genre», effacé par la récriture, tout comme la dimension lyrique, puissante et onirique de l'évocation de l'amour physique, peutêtre jugée trop loin des attentes créées par la nature autobiographique du texte.

Catherine Viollet a été emportée en septembre 2014, alors qu'elle préparait ce volume avec Danielle Constantin. Un texte liminaire, rédigé par ses collègues et amis, rappelle qui fut cette chercheuse hors norme, pionnière des études à la croisée du féminisme, du genre, de la sexualité, grâce notamment, mais non exclusivement, à son travail essentiel sur Violette Leduc. À la fin du volume, sa vaste bibliographie, compilée par Danielle Constantin et Rodolphe Baudin, constitue une précieuse contribution aux études génétiques, et permettra aux lecteurs de découvrir une œuvre critique qui aura renouvelé, de manière éclairante et audacieuse, l'approche d'auteur.e.s trop souvent ignorés par le champ universitaire, en raison précisément de problématiques liées à leur genre ou leur sexualité.

Pierre-Marc de Biasi et Anne Herschberg Pierrot (dir.), L'Euvre comme processus, Paris, CNRS Éditions, 2017, 587 p.

\section{Compte rendu par Grégory} Jouanneau-Damance*

Le recueil collectif L'Euvre comme processus, publié en 2017 aux Éditions du CNRS, sous la direction de Pierre-Marc de Biasi et d'Anne Herschberg Pierrot, réunit les actes du congrès international de critique génétique, qui s'est tenu en septembre 2010 au centre culturel de Cerisy. Dans la lignée des deux précédentes rencontres, organisées en 1987 et 1998, il a pris la forme d'un «travail collectif de bilan et de prospective», et s'est efforcé de présenter un état des lieux de la recherche, de même qu'une synthèse des avancées de cette discipline. Aussi L'Euvre comme processus réunit-il près de cinquante interventions d'universitaires d'une douzaine

* Université Paris VIII, Université Paris X. 